\title{
Optical link ASICs for LHC upgrades
}

\author{
K.K. Gan ${ }^{1}$, H.P. Kagan, R.D. Kass, J.R. Moore, D.S. Smith
}

The Ohio State University

Department of Physics

Columbus, $\mathrm{OH} 43210$, USA

E-mail: ganemps.ohio-state.edu

We have designed several ASICs for possible applications in a new ATLAS pixel layer for the first phase of the LHC luminosity upgrade. The ASICs include a high-speed driver for the VCSEL, a receiver/decoder to decode the signal received at the PIN diode to extract the data and clock, and a clock multiplier to produce a higher frequency clock to serialize the data for transmission. These chips were designed using a $130 \mathrm{~nm}$ CMOS process to enhance the radiation-hardness. We have characterized the fabricated chips and the submission has been mostly successful. We irradiated the chips with $24 \mathrm{GeV} / \mathrm{c}$ protons at CERN to a dosage of 70 Mrad. We observed no significant degradation except the driver circuit in the VCSEL driver fabricated using the thick oxide process in order to provide sufficient voltage to drive a VCSEL. The degradation is due to the large threshold shifts in the PMOS transistors used.

European Physical Society Europhysics Conference on High Energy Physics EPS-HEP 2009,

Krakow , Poland

July 16-22, 2009

$1 \quad$ Speaker 


\section{Introduction}

The Large Hadron Collider (LHC) at CERN will be upgraded in two phases, resulting in ten time higher luminosity. The detectors are expected to be exposed to a similar increase in radiation. We have designed several ASICs for possible applications in a new pixel layer for the ATLAS experiment in the first phase of the luminosity upgrade. The ASICs were fabricated using a $130 \mathrm{~nm}$ CMOS 8RF process [1] and include two VCSEL drivers $(640 \mathrm{Mb} / \mathrm{s}$ and 3.2 $\mathrm{Gb} / \mathrm{s}$ ), a PIN receiver with a clock and data recovery circuit capable of operation at 40,160 , or $320 \mathrm{Mb} / \mathrm{s}$, and two clock multipliers designed to operate at $640 \mathrm{Mb} / \mathrm{s}$. The clock multiplier is needed to produce a higher frequency clock to serialize the data for transmission. All circuitry within the test chip was designed following test results and guidelines from CERN on radiation tolerant design for the process used [2]. We summarize below the results from the characterization, including the irradiation with $24 \mathrm{GeV}$ protons at CERN. All chips tested were packaged for the irradiation and therefore we experienced some speed degradation due to the added stray capacitance of the packaging.

\section{VCSEL driver chip}

The two VCSEL driver chips have similar architecture, one optimized for $640 \mathrm{Mb} / \mathrm{s}$ with higher drive current and the other for $3.2 \mathrm{~Gb} / \mathrm{s}$ with lower current. Each consists of a $1.5 \mathrm{~V}$ LVDS receiver circuit, a 1.5 to $2.5 \mathrm{~V}$ logic converter circuit, and a $2.5 \mathrm{~V}$ VCSEL driver circuit. Both VCSEL drivers allow for adjustable bias and modulation currents and contain circuitry to reduce switching noise on the power supply lines. We used a $2.5 \mathrm{~V}$ supply for the driver circuitry because most commercially available VCSELs require bias voltages greater than $2 \mathrm{~V}$ to produce suitable output optical power. The driver portion of the chips was therefore designed using the thick oxide transistors instead of thin oxide transistors for all other circuits. Previous results from CERN have shown that thin oxide transistors designed using conventional layout techniques exhibit radiation tolerance suitable for SLHC applications. However, conventionally designed thick oxide transistors are not suitably radiation tolerant. All of the thick oxide transistors were therefore designed using an enclosed structure.

The performance of the four packaged chips was satisfactory up to $1 \mathrm{~Gb} / \mathrm{s}$. The performance at higher speeds could not be sufficiently evaluated due to the packaging parasitics. In the irradiation, each chip was connected to a $25 \Omega$ resistor instead of a VCSEL to allow testing of the degradation of the chip alone. The duty cycle of the output signal and the current consumption of the LVDS receiver remained constant during the irradiation. However, we observed significant decrease in the current consumption of the VCSEL driver circuit and the output drive current. Post-irradiation analysis of the IV characteristic of a PMOS transistor fabricated in the thick oxide technology indicates a significant threshold shift and the effect can be emulated in a simulation. The shift is significantly smaller in the NMOS transistors. The present current mirror in the driver circuit uses both types of transistors and thus is sensitive to the different threshold shifts. The plan is to use PMOS transistors only in the future design. 


\section{PIN receiver and decoder chip}

The PIN receiver/decoder contains a trans-impedance amplifier, limiting amplifier, biphase mark (BPM) decoder with clock recovery, and LVDS drivers to send the decoded data and recovered clock off chip. The clock and data are recovered using a delay locked loop with networks of switchable capacitors in the delay stages allow for the three operating frequencies, 40, 160, and $320 \mathrm{Mb} / \mathrm{s}$. However, due to insufficient time to optimize the design, the chips operate at somewhat lower speed and require higher threshold currents to achieve a low bit error rate (BER). Other than these two limitations, the performance of the chips is satisfactory, including clock jitter, duty cycle, rise/fall time, and high/low levels of the LVDS drivers.

For the irradiation, four chips were tested in each of the two setups, one was purely electrical and the other involved a PIN diode so that we could decouple the electrical and optical degradations. The decoded data were transmitted to the control room using $20 \mathrm{~m}$ of coax. In the first setup, $40 \mathrm{Mb} / \mathrm{s}$ BPM signals were transmitted over $20 \mathrm{~m}$ of coax to the chips. In the second setup, we sent $40 \mathrm{Mb} / \mathrm{s}$ BPM signal via a fiber to a PIN diode coupled to a chip. We observed single event upset during the spill but observed no degradation in the threshold for $\sim 1$ error/s. The BER decreased with larger PIN current and was higher for a chip coupled to a PIN diode as expected. The current consumption was constant during the irradiation.

\section{Clock multiplier}

The clock multiplier circuits on the test chip consist of charge pump/ring oscillator phase locked loops (PLL) with dividers in the feedback loop. One circuit performs a frequency multiplication of 16 and the other a multiplication by 4, both yielding a $640 \mathrm{MHz}$ clock. All four multipliers packaged for the irradiation functioned well with clock jitter of $<8$ ps or $0.5 \%$. During the irradiation, we observed that the clocks of two chips lost lock and power cycling was needed to resume operation at $640 \mathrm{MHz}$. This problem is not yet understood.

\section{Summary}

We have designed prototype ASICs using the $130 \mathrm{~nm}$ process to enhance the radiationhardness. We observed no significant performance degradation up to a dose of $70 \mathrm{Mrad}$ except in the VCSEL driver. This is due a large threshold shift in the PMOS transistors fabricated in the thick oxide technology for the operation at $2.5 \mathrm{~V}$ to drive the VCSEL.

\section{Acknowledgement}

This work was supported in part by the U.S. Department of Energy under contract No. DE-FG-0291ER-40690. The authors are indebted to M. Glaser for his help with the T7 irradiation facility at CERN.

\section{References}

[1] The VCSEL driver and PIN receiver/decoder is an updated version of the chips designed in the 250 nm process, K. Arms et al., Nucl. Instrum. Methods. A. 554 (2005) 458.

[2] F. Faccio, personal communication, 2007. 